stationäre Behandlung wegen ausgeprägter Symptomatik nötig. Die 1-Jahres-Überlebensrate solcher Patienten liegt ohne Transplantation bei $40 \%$.

Pathophysiologisch werden bei der hepatischen Enzephalopathie neben einem gestörten Ammoniakstoffwechsel auch Entzündungsvorgänge im Gehirn und eine gestörte Autoregulation des zerebralen Blutflusses diskutiert.

Bei der Therapie der hepatischen Enzephalopathie steht die Ausschal- tung der auslösenden Faktoren - soweit möglich - im Vordergrund. Dazu gehören die Therapie von Obstipation, gastrointestinalen Blutungen, Infektionen und die Vermeidung von Proteinexzessen bei der Ernährung.

Darüber hinaus empfiehlt sich eine Eiweißzufuhr von $1-1,5 \mathrm{~g} / \mathrm{kg}$ KG pro Tag. Eine weitere Reduktion der täglichen Proteinmenge führt zu keiner Verbesserung der hepatischen Enzephalopathie. Durch nicht resorbierbare
Disaccharide wie z. B. Laktulose kann die intestinale Ammoniakproduktion gehemmt werden. Gleiches gilt für nicht resorbierbare Antibiotika wie Neomycin oder Rifaximin. Ein weiterer medikamentöser Therapieansatz ist Ornithin-Aspartat, ein Dimer aus zwei Aminosäuren. Für Probiotika liegen bisher keine überzeugenden Studienergebnisse vor.

Dr. med. Peter Stiefelhagen .

\title{
Lebertransplantation
}

\section{Die einzige kurative Therapie}

\begin{abstract}
Die Lebertransplantation ist bei Patienten mit fortgeschrittener Leberzirrhose die einzige kurative Therapieoption. Leider können angesichts des eklatanten Spendermangels nicht alle Patienten, die profitieren würden, transplantiert werden.
\end{abstract}

— „Die Verfeinerung der chirurgischen Technik, Fortschritte in der Anästhesie und Intensivmedizin, der Immunsuppression und der antimikrobiellen Therapie haben zu einer signifikanten Verbesserung der Ergebnisse nach Lebertransplantation geführt", sagte Prof. Alfred Königsrainer, Tübingen.

Indiziert ist die Transplantation bei allen Patienten mit einer dekompensierten chronischen Hepatopathie und einer geschätzten Lebenserwartung von weniger als einem Jahr, soweit keine Kontraindikationen wie eine systemische Tumorerkrankung oder ein lokal fortgeschrittenes hepatozelluläres Karzinom, ein fortbestehender Alkoholismus oder Drogenkonsum vorliegen. Wegen des Organmangels wird zunehmend auch die Möglichkeit einer Leberlebendspende diskutiert.

Die 1- und 5-Jahres-Überlebensraten bei allen transplantierten Patienten, unabhängig von der Indikation, liegen heute bei 85 bzw. 70\%. Die bes-

Transplantation einer neuen Leber. ten Langzeitergebnisse haben Patienten mit einer cholestatischen Lebererkrankung als Grunderkrankung, gefolgt von den Stoffwechselerkrankungen, der alkoholischen Zirrhose, den posthepatitischen Zirrhosen und den Tumoren.

\section{Rezidive, Infektionen und Organversagen}

Ein besonderes Problem bei Patienten mit einer chronischen Hepatitis B oder C ist die Rezidivinfektion des transplantierten Organs. Während das Rezidiv in der Hepatitis-B- Virus-Zirrhose durch eine passive Immunisierung und Virostatika bei einem Großteil der betroffenen Patienten heute verhindert werden kann, ist das Rezidiv bei der HCV-Zirrhose fast 100\%.

Neben dem Rezidiv der Grunderkrankung gibt es noch andere Ursachen für ein Transplantatversagen.

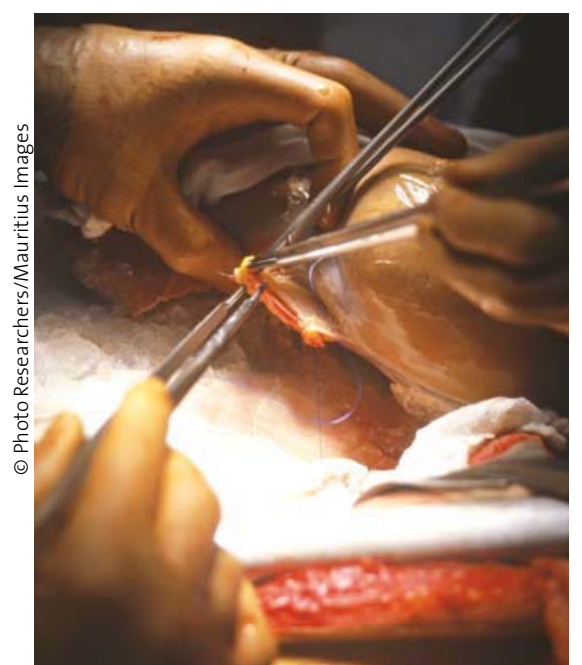

Dazu gehören chronische Abstoßungsreaktionen und Infektionen. „Infektionskrankheiten sind in ca. $70 \%$ der Fälle die Ursache für das Versterben der Patienten nach einer Lebertransplantation“, so Königsrainer.

In der Immunsuppression wurden in den letzten Jahren große Fortschritte erzielt, dennoch ist nach wie vor mit gravierenden Nebenwirkungen zu rechnen. Dazu gehören Niereninsuffizienz, arterielle Hypertonie, Glukoseintoleranz bzw. Diabetes mellitus, Hyperlipoproteinämie, Osteoporose und neurologische Symptome.

\section{Frühe Vorstellung sinnvoll}

Grundsätzlich empfiehlt es sich, so Königsrainer, Patienten mit einer chronisch progredienten Lebererkrankung früh in einem Lebertransplantationszentrum vorzustellen. Gerade die Option einer Leberlebendspende-Transplantation sollte möglichst ohne Zeitdruck langfristig in der Familie bzw. im engsten Freundeskreis diskutiert werden. Dabei müssen die Vorteile für den Empfänger gegenüber dem Risiko des Spenders abgewogen werden. Auch bei sorgfältigster Selektion des Spenders besteht ein Mortalitätsrisiko von 0,2\% im Rahmen der Lebendspende.

Dr. med. Peter Stiefelhagen .

- Quelle: XIII. Gastroenterologie-Seminarwoche Titisee, 27.2. - 3.3.2010 